\title{
Increased plasma soluble CD40 ligand concentrations in systemic sclerosis and association with pulmonary arterial hypertension and digital ulcers
}

\author{
Y Allanore, D Borderie, C Meune, H Lemaréchal, S Weber, O G Ekindjian, A Kahan
}

Ann Rheum Dis 2005;64:481-483. doi: 10.1136/ard.2003.020040

\begin{abstract}
Background: Increased expression of CD4OL has been reported on activated $\mathrm{CD} 4+\mathrm{T}$ lymphocytes in systemic sclerosis. CD4OL can be expressed in soluble form (sCD4OL). Objective: To compare sCD4OL concentrations in patients with systemic sclerosis and healthy controls.

Methods: Quantitative sandwich ELISA was used to measure plasma $\mathrm{SCD} 40 \mathrm{~L}$ in systemic sclerosis $(n=50)$ and matched healthy controls $(n=20)$. Patients with systemic sclerosis had limited cutaneous disease (29), digital ulcers (14), pulmonary arterial hypertension (PAH) (10), pulmonary fibrosis on CT (23), positive anti-Scl70 (14), and anti-centromere antibodies (10). Calcium channel blockers were discontinued 72 hours before measurements.

Results: Median (range) sCD4OL concentration (pg/ml) was higher in systemic sclerosis than in controls (495 (10 to 7720) $v 79$ (50 to 118); $p=0.003$ ), in limited cutaneous disease $v$ diffuse disease (620 (20 to 7720) v 250 (10 to 2690); $\mathrm{p}=0.005)$, in patients with digital ulcers $v$ those without (1430 (36 to 7720$) \vee 370$ (10 to 2320); $p=0.002)$, and in those with PAH $v$ those without (995 (15 to 3850) v 400 (10 to 7720); $p=0.048)$. sCD40L correlated with pulmonary arterial pressure estimated by Doppler echocardiography $(r=0.41 ; p=0.005)$.

Conclusions: The soluble form of $\mathrm{CD} 40 \mathrm{~L}$ is increased in plasma in systemic sclerosis and may be associated with vascular complications of the disease.
\end{abstract}

\footnotetext{
$\mathrm{T}$
} he CD40/CD154 interaction is pivotal in the induction of humoral and cellular immune responses. CD40 and its ligand are transmembrane glycoproteins that belong to the tumour necrosis factor (TNF) receptor superfamily. ${ }^{1}$ Activated CD4 $\mathrm{T}$ cells express the CD40 ligand (CD40L) which can engage CD40 on the B cell surface; this triggers a cascade resulting in expression of surface accessory and adhesion molecules. ${ }^{1}$ However, CD40L can be cleaved from the cell surface, releasing a soluble CD40L (sCD40L) which is biologically active. SCD40L is increased in systemic lupus erythematosus, ${ }^{2}$ rheumatoid arthritis and associated vasculitis, ${ }^{3}$ and mixed connective tissue disease. ${ }^{4}$

Systemic sclerosis is a connective tissue disease characterised by vascular involvement with generalised microangiopathy and serum autoantibodies, culminating in systemic fibrosis. Although the pathophysiology remains unclear, early vascular lesions result from interaction between endothelium and activated lymphocytes with the involvement of adhesion molecules and cytokines.

Increased expression of the CD40L molecule has previously been reported on the surface of activated $\mathrm{T}$ cells in systemic sclerosis. ${ }^{5}$ Our aim in this study was to investigate plasma
SCD40L in patients with systemic sclerosis compared with healthy controls, and to look for an association of this marker with visceral involvement.

\section{METHODS}

\section{Study population}

The study was prospective. We recruited successive patients with systemic sclerosis who had been admitted to hospital and were scheduled for systematic follow up. Systemic sclerosis was classified as limited or diffuse cutaneous according to the criteria of LeRoy et al. ${ }^{6}$ The exclusion criteria were the impossibility of stopping vasodilator treatment, use of immunosuppressive therapy, and association with severe diseases (cardiac or pulmonary failure, cancer, gangrene). Three months of stable current treatment was a requirement for inclusion; treatment with prednisone at a dose less than $10 \mathrm{mg} /$ day was allowed. Patients were asked to stop taking calcium channel blockers three days before the studies.

Laboratory tests included blood cell count, Westergren erythrocyte sedimentation rate, $\mathrm{C}$ reactive protein levels, serum creatinine, and antinuclear, anticentromere (indirect immunofluorescence on Hep-2 cells), antitopoisomerase I (counter immunoelectrophoresis), and antiphospholipid (enzyme linked immunosorbent assay (ELISA)) antibody levels. The modified Rodnan skin score was used for skin assessment, ${ }^{7}$ and pulmonary involvement was assessed by computed tomography (CT), forced vital capacity (FVC), and carbon monoxide diffusion capacity (DLCO/haemoglobin ratio). Left ventricular function was assessed by radionuclide ventriculography, and systolic pulmonary artery pressure (PAPs) by Doppler echocardiography. For the purpose of this study, PAH was defined as a PAPs above $40 \mathrm{~mm} \mathrm{Hg}$ with no severe pulmonary fibrosis (FCV $>50 \%$ ). The controls were 20 healthy subjects (18 women and two men) from the laboratory staff, with a mean (SD) age of 49.6 (9.0) years.

\section{Assay of soluble CD40L}

According to previous recommendations, ${ }^{8}$ we used plasma samples. Blood samples $(10 \mathrm{ml})$ were collected in tubes containing ethylenediamine-tetraacetic acid. They were centrifuged at $3000 \times \mathrm{g}$ for 10 minutes within 30 minutes of collection. After separation, plasma were stored at $-80^{\circ} \mathrm{C}$ until use. Plasma sCD40L were measured by ELISA (R\&D systems Abingdon, UK). Briefly, diluted (1:5) plasma samples were applied to 96-well plates precoated with a polyclonal antibody specific for CD40 ligand and incubated for two hours. After washing, a horseradish peroxidase linked polyclonal antibody specific for CD 40 ligand was added to the wells and incubated. Subsequently, plates were washed

Abbreviations: DCLO, lung diffusing capacity for carbon monoxide; $\mathrm{PAH}$, pulmonary artery hypertension; PAPs, systolic pulmonary artery pressure 
Table 1 Characteristics of 50 patients with systemic sclerosis

\begin{tabular}{ll}
\hline Cutaneous form of disease (limited/diffuse) (n (\%)) & $29(58 \%) / 21$ \\
& $(42 \%)$ \\
Current digital ulcers (n (\%) patients) & $14(28 \%)$ \\
Pulmonary arterial pressure (mm Hg) (mean (SD) & $33(8)(16$ to 59) \\
(range)) & $10(20 \%) / 47(5)$ \\
PAPs $\geqslant 40 \mathrm{~mm} \mathrm{Hg}$ (n (\%)/mean (SD) pressure) & $25(50 \%)$ \\
Pulmonary fibrosis (CT scan) (n (\%) patients) & $12(24)$ \\
Forced vital capacity <75\% of normal values (n (\%)) & $12(40)$ \\
Decreased CO diffusing capacity (n (\%) patients) & $20(40)$ \\
Left ventricular ejection fraction (mean (SD)) & $63(8)$ \\
Antinuclear antibodies ( $\geqslant 1 / 160)$ (n (\%)) & $43(86)$ \\
Anti-Scl70 antibodies/anticentromere antibodies & $14 / 10$ \\
(n/n) & \\
Anticardiolipin antibodies (lgG or lgM $>15$ IU) & $8(16)$ \\
(n (\%)) & $78(13)$ \\
Serum creatinine ( $\mu$ mol/I) (mean (SD)) & $16.5(15.4)$ \\
ESR (mm/hour) (mean (SD)) & $6.7(5.9)$ \\
C reactive protein (mg/l) (mean (SD)) & $20(7.4(2))$ \\
Ongoing low dose prednisone (n (mean (SD) & \\
mg/day) & 9 \\
Ongoing ACE inhibitor treatment (n) & 34 \\
Ongoing antiaggregant treatment (n) & \\
\hline
\end{tabular}

$\mathrm{ACE}$, angiotensin converting enzyme; $\mathrm{CO}$, carbon monoxide; $\mathrm{CT}$, computed tomography; ESR, erythrocyte sedimentation rate; PAPs, systolic pulmonary artery pressure

and antibody binding determined by colorimetry using 3,3'5,5'-tetramethylbenzidine substrate. Absorbance was read using a Dynatech MR 5000 microplate reader (Dynex Technology) at $450 \mathrm{~nm}$ within 30 minutes. According to the manufacturer, intra-assay precision was $5.1 \%$ for $430 \mathrm{pg} / \mathrm{ml}$ $(\mathrm{n}=20)$ and $5.4 \%$ for $2638 \mathrm{pg} / \mathrm{ml} \quad(\mathrm{n}=20)$; interassay precision was $6.4 \%$ for $437 \mathrm{pg} / \mathrm{ml}(\mathrm{n}=40)$ and $6.2 \%$ for $2612 \mathrm{pg} / \mathrm{ml}(\mathrm{n}=40)$.

\section{Statistical analysis}

Data were analysed with the following non-parametric tests: Mann-Whitney and Wilcoxon tests for comparison of groups and Spearman's rank correlation test for assessment of the relation between quantitative variables. Probability $(p)$ values

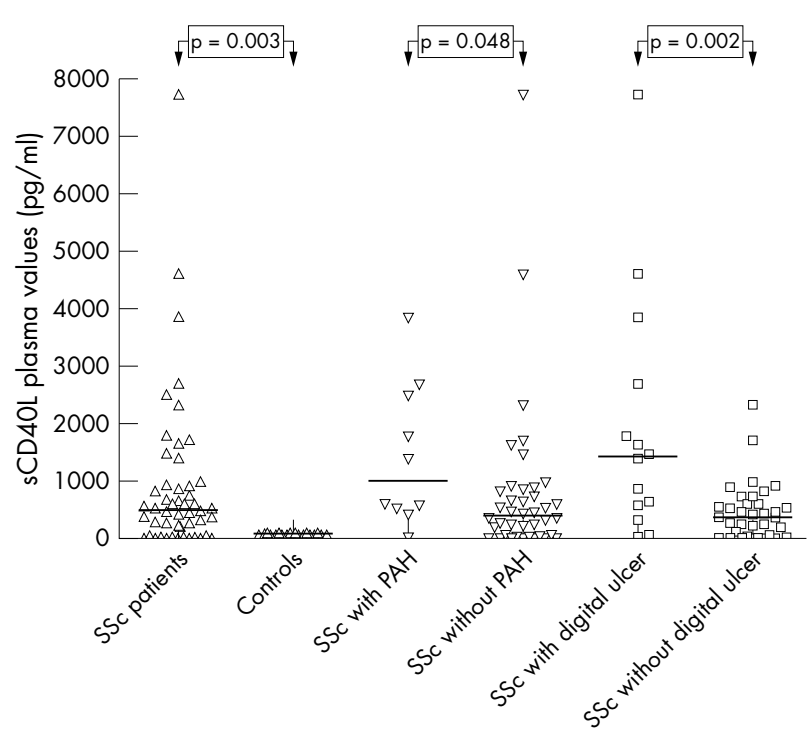

Figure $1 \mathrm{sCD} 40 \mathrm{~L}$ (individual plasma concentrations $(\mathrm{pg} / \mathrm{ml}$ ) and median values) in patients with systemic sclerosis (SSc, $\mathrm{n}=50$ ) and controls ( $n=20)$; in SSc patients with $(n=14)$ and without digital ulcers $(n=36)$; and in SSc patients with $(n=10)$ and without $(n=40)$ pulmonary arterial hypertension (PAH). less than 0.05 were considered significant. All quantitative data are expressed as median (range).

\section{RESULTS}

We included 50 successive patients with systemic sclerosis. There were 44 women and six men, mean (SD) age, 57 (11) years, range 34 to 80 . Mean disease duration was 7 (7) years; in 18 patients disease duration was less than three years. The clinical and laboratory data for these patients are presented in table 1.

The median (range) concentration of SCD40L was higher in the patients with systemic sclerosis than in the healthy controls, at 495 (10 to 7720$) \quad v 79$ (50 to 118 ) pg/ml $(\mathrm{p}=0.003$; Mann-Whitney $\mathrm{U}$ test; fig 1$)$. Median $\mathrm{sCD} 40 \mathrm{~L}$ was increased in patients with limited cutaneous disease compared with those with diffuse disease, at 620 (20 to 7720) $v 250$ ( 10 to 2690$) \mathrm{pg} / \mathrm{ml}$ ( $\mathrm{p}=0.005$; Mann-Whitney). In the 10 patients with $\mathrm{PAH}$, median sCD40L was higher than in those without PAH, at 995 ( 15 to 3850) $v 400$ ( 10 to 7720 ) pg/ $\mathrm{ml}$ ( $\mathrm{p}=0.048$; Mann-Whitney). CD40L correlated with PAPs estimated by Doppler echocardiography $(r=0.41 ; \mathrm{p}=0.005$, Spearman's test). Patients with systemic sclerosis and digital ulcers had higher concentrations of SCD40L than those without, at 1430 (36 to 7720 ) v 370 (10 to 2320) pg/ml $(\mathrm{p}=0.002$; Mann-Whitney; fig 1$)$. In the subgroup of patients with limited disease $(n=29)$, median sCD40L was higher in patients with PAH $(\mathrm{n}=5)$ than in those without PAH $(\mathrm{n}=24)$, at 2140 (1390 to 3850) v 600 (20 to 7720$) \mathrm{pg} /$ $\mathrm{ml}(\mathrm{p}=0.04)$; and also higher in patients with digital ulcers than in those without: 1390 (36 to 7720) v 509 (20 to 250) pg/ $\mathrm{ml}(\mathrm{p}=0.02$; (Mann-Whitney).

Age, disease duration, concurrent cutaneous skin score, pulmonary fibrosis, carbon monoxide diffusion, autoantibody status, and treatment (antiaggregant, corticosteroids, angiotensin converting enzyme inhibitors) were not associated with SCD40L concentrations.

\section{DISCUSSION}

We report a significant increase in the soluble form of CD40L in the plasma of patients with systemic sclerosis. If the 90th centile of the control concentrations $(107.8 \mathrm{pg} / \mathrm{ml})$ was taken as the upper limit of normal, 37 of the 50 patients (74\%) had increased concentrations. Patients with pulmonary arterial hypertension and digital ulcers had the highest levels of SCD40L.

It has recently been suggested that an abnormal cell mediated response between fibroblasts and T cells in the skin lesions of systemic sclerosis may implicate the CD40-CD40L system. ${ }^{9}$ In our study, we report higher values of sCD40L in patients with limited cutaneous disease than in those with diffuse disease, and sCD40L concentrations did not correlate with the skin score. Our results do not support the view that sCD40L reflects fibroblasts activation. It has recently been reported that membrane tumour necrosis factor may be involved in the inhibition of fibroblast synthesis by Th2 lymphocytes ${ }^{10}$; the involvement of CD40L in this phenomenon should be investigated.

We report here that the presence of digital ulcers was associated with SCD40L concentrations in the whole study population and also in the subgroup of patients with limited disease, which was stratified to limit the influence of cutaneous subtype and disease duration on sCD40L levels. Concentrations of sCD40L were significantly higher in patients with PAH. These patients had early and mild PAH, and the finding needs to be confirmed in patients with severe PAH.

Compared with previous studies, our mean (SD) serum concentration of sCD40L in systemic sclerosis (918 (1384) pg/ $\mathrm{ml})$ was lower than in systemic lupus erythematosus (2610 
(2150) $\mathrm{pg} / \mathrm{ml})^{2}$ or mixed connective tissue disease (median $999 \mathrm{pg} / \mathrm{ml})^{4}$ but higher than in rheumatoid arthritis (730 (1.11) $\mathrm{pg} / \mathrm{ml}$ ). ${ }^{3}$ The $\mathrm{sCD} 40 \mathrm{~L}$ values in our patients with systemic sclerosis were also higher than in the healthy controls in those previous studies (mean (SD), 25 (40) pg/ml in serum, ${ }^{2} 170(190) \mathrm{pg} / \mathrm{ml}$ in plasma, ${ }^{3}$ and median $179 \mathrm{pg} / \mathrm{ml}$ in the final study ${ }^{4}$ ). The values in our control group (median, $79 \mathrm{pg} / \mathrm{ml}$; mean (SD), 81 (19)) are in accord with those data. However, differences in the various methods used do not allow strict comparisons between those results and ours; in particular, we used plasma samples with high speed centrifugation to limit all sources of contamination by platelets. ${ }^{8}$

In systemic lupus erythematosus, SCD40L is thought to reflect $\mathrm{T}$ cell activation or a chronic inflammatory condition ${ }^{2}$; however, recent data also suggest a link with antiphospholipid antibodies and platelet activation in this condition. ${ }^{11}$ We could not identify this link in our patients with systemic sclerosis.

CD40L is believed to be deeply involved in the immune response. In systemic sclerosis, T-B collaboration is essential for the autoimmune response ${ }^{12}$; however, we could not determine an association between autoantibody status and sCD40L concentration. Upon ligation with CD40, CD40L is endocytosed and its mRNA expression is downregulated. Although this mechanism is thought to be the principal way in which the CD40-CD40L interaction is regulated, the production of sCD40L could also act as an antagonist in the immune response and may therefore represent a secondary event in systemic sclerosis. ${ }^{12}$

Although CD40-CD40L has been studied intensively in T-B cell activation, CD40L also binds to other CD40 positive cells, such as endothelial cells, leading to the synthesis of adhesion molecule, chemokines, and matrix metalloproteinases. ${ }^{13}$ Ligation of CD40L on various vascular cells contributes to the pathogenesis of atherosclerotic, thrombotic, and inflammatory processes. ${ }^{14}$ Vasculopathy resulting from endothelial cell activation and activated lymphocyte recruitment is a crucial feature of systemic sclerosis, and coagulation abnormalities are well recognised features ${ }^{15}$; investigation of CD40L and SCD40L in these areas is warranted.

\section{Conclusions}

Our study showed increased plasma soluble CD40 ligand concentrations in systemic sclerosis and an association between the increased values and pulmonary arterial hypertension and digital ulcers. Our results, along with those previously reported, ${ }^{59}$ suggest that CD40L signalling may play an important role in this disease.

\section{ACKNOWLEDGEMENTS}

We thank Mrs Carole Desbas for her expert secretarial assistance.
Authors' affiliations

Y Allanore, A Kahan, Rheumatology A, Paris V University, Assistance

Publique Hôpitaux de Paris, Cochin Hospital, Paris, France

D Borderie, $\mathbf{H}$ Lemaréchal, $\mathbf{O}$ G Ekindjian, Biochemistry A, Paris V

University, Cochin Hospital

C Meune, S Weber, Cardiology, Paris V University, Cochin Hospital

Correspondence to: Dr Yannick Allanore, Hôpital Cochin, service de Rhumatologie A, 27 rue du faubourg Saint-Jacques 75014 Paris, France; yannick.allanore@cch.ap-hop-paris.fr

Accepted 6 July 2004

\section{REFERENCES}

1 Vogel LA, Noelle RJ. CD40 and its crucial role as a member of the TNFR family. Semin Immunol 1998; 10:423-34.

2 Vakkalanka RK, Woo C, Kirou KA, Koshy M, Berger D, Crow MK. Elevated levels and functional capacity of soluble CD40 ligand in systemic lupus erythematosus sera. Arthritis Rheum 1999;42:871-81.

3 Tamura N, Kobayashi S, Kato K, Bando H, Haruta K, Oyanagi M, et al. Soluble CD154 in rheumatoid arthritis: elevated plasma levels in cases with vasculitis. J Rheumatol 2001;28:2583-90.

4 Jinnin M, Ihn H, Yazawa N, Asano Y, Yamane K, Tamaki K. Elevated circulating soluble CD40 ligand in patients with mixed connective tissue disease. Clin Rheumatol 2003;22:37-9.

5 Valentini G, Romano MF, Naclerio C, Bisogni R, Lamberti A, Turco MC, et al. Increased expression of CD40 ligand in activated CD4+ lymphocytes of systemic sclerosis patients. J Autoimmun 2000;15:61-6.

6 LeRoy C, Black C, Fleischmajer R, Jablonska S, Krieg T, Medsger TA, et al. Scleroderma (systemic sclerosis). Classification, subsets and pathogenesis. $J$ Rheumatol 1988;15:202-5.

7 Akesson A, Fiori G, Krieg T, van den Hoogen FH, Seibold JR. Assessment of skin, joint, tendon and muscle involvement. Clin Exp Rheumatol 2003;21(3 suppl 29):S5-8

8 Bereczki D, Nagy E, Pal A, Magyar MT, Balla J. Should soluble CD40 ligand be measured from serum or plasma samples? Arterioscler Thromb Vasc Biol 2003;23:1129-30

9 Fukasawa C, Kawaguchi Y, Harigai M, Sugiura T, Takagi K, Kawamoto M, et al. Increased CD40 expression in skin fibroblasts from patients with systemic sclerosis (SSc): role of CD40-CD154 in the phenotype of SSc fibroblasts. Eur J Immunol 2003;33:2792-800.

10 Chizzolini C, Parel Y, De Luca C, Tyndall A, Akesson A, Scheja A, et al. Systemic sclerosis Th2 cells inhibit collagen production by dermal fibroblasts via membrane-associated tumor necrosis factor alpha. Arthritis Rheum 2003;48:2593-604.

11 Ferro D, Pignatelli P, Loffredo L, Conti F, Valesini G, D'Angelo A, et al. Soluble CD154 plasma levels in patients with systemic lupus erythematosus: modulation by antiphospholipid antibodies. Arthritis Rheum 2004; 50:1693-4.

12 Kuwana M, Medsger TA, Wright TM. T and B cell collaboration is essential for the autoantibody response to DNA topoisomerase I in systemic sclerosis. J Immunol 1995; 155:2703-14.

13 Contin C, Pitard V, Itai T, Nagata S, Moreau JF, Dechanet-Merville J. Membrane-anchored CD40 is processed by the tumor necrosis factor-alphaconverting enzyme. Implications for CD40 signaling. J Biol Chem 2003;278:32801-9.

14 Freedman JE. CD40-CD40L and platelet function: beyond hemostasis. Circ Res 2003;92:944-6.

15 Cerinic MM, Valentini G, Sorano GG, D’Angelo S, Cuomo G, Fenu L, et al. Blood coagulation, fibrinolysis, and markers of endothelial dysfunction in systemic sclerosis. Semin Arthritis Rheum 2003;32:285-95. 